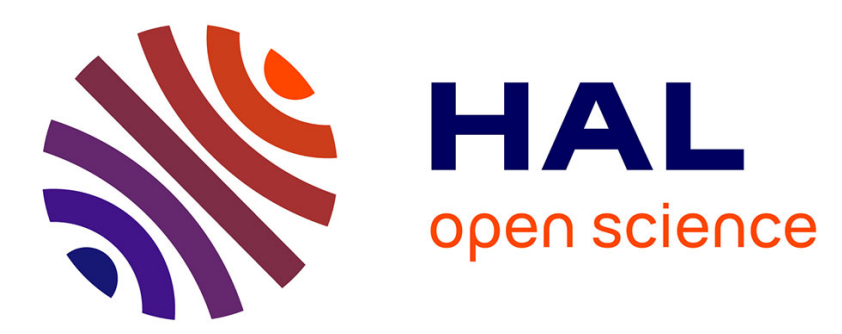

\title{
High resolution spectroscopy of the hydrogen atom. I. Method and experiment
}

\author{
J.C. Garreau, M. Allegrini, L. Julien, F. Biraben
}

\section{To cite this version:}

J.C. Garreau, M. Allegrini, L. Julien, F. Biraben. High resolution spectroscopy of the hydrogen atom. I. Method and experiment. Journal de Physique, 1990, 51 (20), pp.2263-2273. 10.1051/jphys:0199000510200226300 . jpa-00212527

\section{HAL Id: jpa-00212527 https://hal.science/jpa-00212527}

Submitted on 1 Jan 1990

HAL is a multi-disciplinary open access archive for the deposit and dissemination of scientific research documents, whether they are published or not. The documents may come from teaching and research institutions in France or abroad, or from public or private research centers.
L'archive ouverte pluridisciplinaire HAL, est destinée au dépôt et à la diffusion de documents scientifiques de niveau recherche, publiés ou non, émanant des établissements d'enseignement et de recherche français ou étrangers, des laboratoires publics ou privés. 
Classification

Physics Abstracts

$35.10-06.20 \mathrm{~J}-32.80 \mathrm{~K}$

\title{
High resolution spectroscopy of the hydrogen atom. I. Method and experiment
}

\author{
J.C. Garreau $\left({ }^{*}\right)$, M. Allegrini $\left({ }^{* *}\right)$, L. Julien and F. Biraben \\ Laboratoire de Spectroscopie Hertzienne de l'ENS $\left({ }^{* * *}\right)$, Université Pierre et Marie Curie, 4 place \\ Jussieu, Tour 12, E01 75252 Paris Cedex 05, France
}

(Received 2 February 1990, revised 15 June 1990, accepted 27 June 1990)

\begin{abstract}
Résumé. - Pour mesurer la constante de Rydberg $R_{\infty}$ avec une très grande précision, nous avons fait une expérience d'absorption à deux photons sans effet Doppler sur les atomes d'hydrogène et de deutérium. Nous avons obtenu une nouvelle valeur de $R_{\infty}$ qui est en excellent accord avec les autres mesures récentes, et la plus précise à ce jour. Nous décrivons ici en détail la méthode et l'appareillage utilisés. Un jet d'atomes d'hydrogène métastables est excité vers les états de Rydberg $n \mathrm{~S}$ et $n \mathrm{D}(n \geq 8)$ dans une transition à deux photons. La géométrie expérimentale a été choisie de façon à rendre colinéaires le jet atomique et les faisceaux laser. Avec ce montage, nous avons observé des résonances très étroites avec une largeur relative inférieure a $10^{-9}$. Parmi toutes les transitions observées, nous avons selectionné trois transitions (2S-8D, 2S-10D et 2S-12D) dans l'hydrogène et le deutérium pour déterminer $R_{\infty}$.
\end{abstract}

\begin{abstract}
In order to determine the Rydberg constant $R_{\infty}$ to a very high accuracy, we have performed a Doppler-free two-photon absorption experiment on atomic hydrogen and deuterium. We have obtained a new value of $R_{\infty}$ which is in excellent agreement with other recent measurements and the most precise one at the present time. Here we give a detailed report on the method and apparatus we have used. A metastable beam of $2 \mathrm{~S} \mathrm{H}$ and $\mathrm{D}$ atoms has been prepared and laser excited to Rydberg $n S$ and $n \mathrm{D}$ levels $(n \geq 8)$ in a two-photon transition. The experimental geometry has been carefully designed to make the atomic beam collinear with the two counterpropagating laser beams. With this set-up we have observed very narrow resonances, with relative linewidths smaller than $10^{-9}$. Among all the transitions investigated we have selected three transitions $(2 \mathrm{~S}-8 \mathrm{D}, 2 \mathrm{~S}-10 \mathrm{D}$ and 2S-12D) in hydrogen and deuterium to determine $R_{\infty}$.
\end{abstract}

(*) Present address: C.N.E.T., 196 Av. Henri Ravera, 92220 Bagneux, France.

$\left(^{* *}\right)$ Permanent address: Dipartimento di Fisica, Università di Pisa, Piazza Torricelli 2, 56100 Pisa, Italy.

$\left({ }^{* * *}\right)$ Laboratoire de l'Ecole Normale Supérieure et de l'Université Pierre et Marie Curie associé au CNRS (URA 18). 


\section{Introduction.}

In a recent paper [1], we have reported the wavelength measurement of the three transitions $2 S$ 8D, 2S-10D and 2S-12D in atomic hydrogen and deuterium. These measurements lead to a new determination of the Rydberg constant which is at present the most precise one. This paper is the first of a series of three, hereinafter referred to as paper I, II and III respectively, in which we give a complete description of the method used to obtain this result. In this paper (I), we present the principle of our method and we describe the experimental set-up which allowed us to observe very narrow signals. Paper II will be devoted to the signal analysis with special regard to the comparison of calculated line profiles with the experimental curves. Paper III will give the details of the absolute wavelength measurement procedure and the Rydberg constant determination.

The Rydberg constant $R_{\infty}$ is one of the fundamental physical constants which is important to many areas of physics and chemistry $[2,3]$. It is simply related to the other fundamental quantities $m$ (electron mass), $e$ (electron charge), $h$ (Planck constant) and $c$ (velocity of light):

$$
R_{\infty}=\frac{m e^{4}}{8 \epsilon_{0}^{2} h^{3} c}
$$

and it is one of the most accurately measured. There are several reasons for the continuous effort to improve its precision. Indeed it plays a key role in all calculations of atomic and molecular levels and in the least-squares fit of the fundamental constant values where it is used as an auxiliary constant (i.e. with a fixed value not subjected to adjustment) $[4,5]$. A very precise value of $R_{\infty}$ is required to test QED calculations of simple systems; two examples are the determination of the $\mathrm{H}$ 1S ground state Lamb shift and the comparison between measured and calculated frequency of the positronium $1^{3} S_{1}-2^{3} S_{1}$ transition. Since $R_{\infty}$ can in principle be measured from the frequency of any of the atomic hydrogen transitions, it provides a severe test of the $1 / r$ dependence of the Coulomb potential which may prove the existence of the "fifth force" [6] . Measurements of $R_{\infty}$ with equal precision in different spectral regions would permit the use of the hydrogen atom as a wavelength-frequency standard in the optical domain $[7,8]$.

During the last ten years several measurements of $R_{\infty}$ have been performed on atomic hydrogen and deuterium using the refined techniques of high resolution laser spectroscopy. Early discrepancies are now settled and all recent results are in excellent agreement $[1,9-13]$. The accuracy of $R_{\infty}$ has been improved from 3 parts in $10^{8}$, achieved in the first laser experiment on hydrogen [14], to a few parts in $10^{10}$. The limit is now given by the accuracy of the present wavelength-frequency standard in the visible, namely the $I_{2}$-stabilized He-Ne laser [15] .

\section{Method.}

The energy of the hydrogen atom levels is strictly proportional to the Rydberg constant; thus a direct measurement of the wavelength or frequency of an atomic $\mathrm{H}$-transition is in principle equivalent to a measurement of $R_{\infty}$. Transitions involving low values of the principal quantum number $\mathrm{n}$ lie in the optical domain and $R_{\infty}$ is in this respect the natural unit of optical frequencies. It is evident that the accuracy of $R_{\infty}$ depends upon the measurement of the absolute frequency of the hydrogen spectral line under investigation. Both the lineshape and linewidth of the atomic transition are therefore crucial for the final precision of the $R_{\infty}$ value.

Figure 1 is a schematic representation of the transitions investigated in our experiment as well as in other recent experiments performed in the optical domain. The most straightforward way is to study the 2S-3P and 2S-4P Balmer lines because they correspond to direct one-photon transitions. 
Indeed they have been thoroughly investigated at Yale in a set of neat and elegant experiments $[16,10,11]$ which are the equivalent to the early microwave experiments $[17]$ designed for the Lamb shift measurements. The linewidths of these resonances reflect the natural width of the P-states involved (30 MHz and $12 \mathrm{MHz}$ respectively) which is the main limiting factor on the precision of $R_{\infty}$ measured in these experiments.

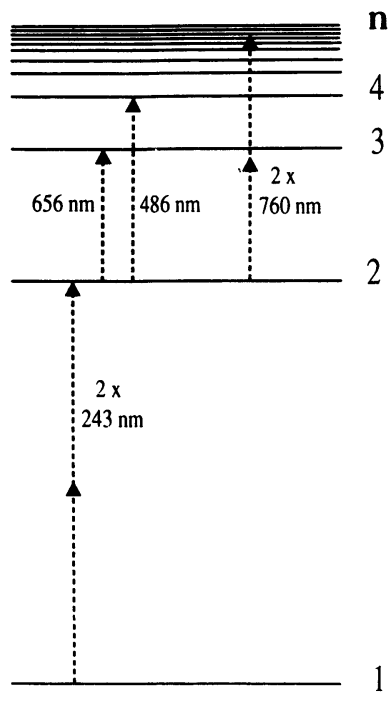

Fig. 1. - Schematic energy diagram of atomic hydrogen.

Of special nature and interest is the 1S-2S two-photon transition which has been extensively investigated by three different groups (Stanford-Münich, Southampton and Oxford). This is one of the sharpest lines available to spectroscopists; due to the $1.3 \mathrm{~Hz}$ linewidth of the metastable $2 \mathrm{~S}$ level, the relative width of the $1 \mathrm{~S}-2 \mathrm{~S}$ transition is $5 \times 10^{-16}$ only. However, the $1 \mathrm{~S}-2 \mathrm{~S}$ transition is not the ideal choice for a Rydberg constant measurement because it is affected by the uncertainty in the 1S Lamb shift. Therefore the 1S-2S transition wavelength measurement provides a value of the Rydberg constant within the uncertainty of the computed Lamb shift, or equivalently allows to determine the 1S Lamb shift, the value of the Rydberg constant being taken from other sources.

The way we have chosen to get very sharp lines is to investigate the two-photon transitions from $2 S$ to $n S$ and $n \mathrm{D}$ states $(n \geq 8)$ in atomic hydrogen and deuterium [18]. The natural width of these levels decreases as $n^{-3}$ and for the $10 \mathrm{D}$ state for example is $\approx 300 \mathrm{kHz}$. First-order Doppler effect has been eliminated by the two-photon absorption technique [19] , based on the interaction of the atom with a standing wave resulting from the superposition of two counterpropagating laser beams of the same frequency. This technique, like other non-linear processes, requires high laser power density which is responsible for broadening and shift of the transitions. However, these effects can be corrected for very precisely, as will be described in paper II. In order to avoid collisional broadening the metastable atoms are produced in a beam which is an important part of our apparatus. Moreover we have used a geometry which makes the metastable beam collinear with the two laser beams so that laser-atom interaction time is long and the resulting line broadening reduced.

An advantage of working with the $2 S-n S$ and $2 S-n D$ transitions is that the Lamb shift of the 
metastable $2 S$ state has been already measured very precisely $[20,21]$. For the Rydberg states it is very small and calculated to high precision. Indeed our method to determine $R_{\infty}$, once we have measured the frequency or wavelength of the $2 S_{1 / 2}-8 D_{5 / 2}, 2 S_{1 / 2}-10 D_{5 / 2}$ and $2 S_{1 / 2}-$ $12 \mathrm{D}_{5 / 2}$ transitions of atomic hydrogen, takes advantage of the fact that the $2 \mathrm{~S}$ Lamb shift is known with an accuracy of few $\mathrm{kHz}$. We have used the Pal'chikov et al. [20] experimental value of the $2 \mathrm{~S}_{1 / 2}-2 \mathrm{P}_{1 / 2}$ energy interval and our measurement of the $2 \mathrm{~S}_{1 / 2}-n \mathrm{D}_{5 / 2}(n=8,10,12)$ transition wavelengths to get the frequencies of the corresponding $2 \mathrm{P}_{1 / 2}-n \mathrm{D}_{5 / 2}$ transitions. By comparing the theoretical and experimental values of this last energy interval, for which $R_{\infty}$ is a scaling factor, we determine $R_{\infty}$. Therefore the precision of this measurement is not affected by the QED corrections on the $2 S$ energy, but only by those on the $2 \mathrm{P}$ and $n \mathrm{D}$ levels $(n=8,10,12)$ which are at least a factor 50 smaller. The overall effect of QED corrections, $2 S$ Lamb shift precision and electron-to-proton mass ratio gives at the present time an intrinsic limit (i.e. not related to the experiment) of $1.9 \times 10^{-11}$. For the determination of $R_{\infty}$ we have also used three transitions of atomic deuterium for which the $2 S$ Lamb shift has not been measured with such high accuracy as in hydrogen. Nevertheless we have used the same procedure except for the fact that we have been forced to use a theoretical value for the 2 S Lamb shift $(1057.229 \mathrm{MHz})$, which we have calculated using the formula given by Erickson and Grotch [22] . As we will see in paper III, the six values of $R_{\infty}$ obtained from the three hydrogen and the three deuterium transitions are consistent each with others. Thus this result is an indirect test of the agreement between calculated and measured values of the $2 \mathrm{~S}$ Lamb shift.

\section{Apparatus.}

The basic apparatus was already used in 1986 for a first measurement of $R_{\infty}$ by two-photon spectroscopy of hydrogen Rydberg states [9]. For the present experiment several improvements have been made designed to reduce the linewidth and to measure the absolute wavelength of the observed transitions with the precision of the wavelength standard in the visible region. The apparatus consists of four main parts sketched in figure $2:$ the atomic beam, the laser source, the

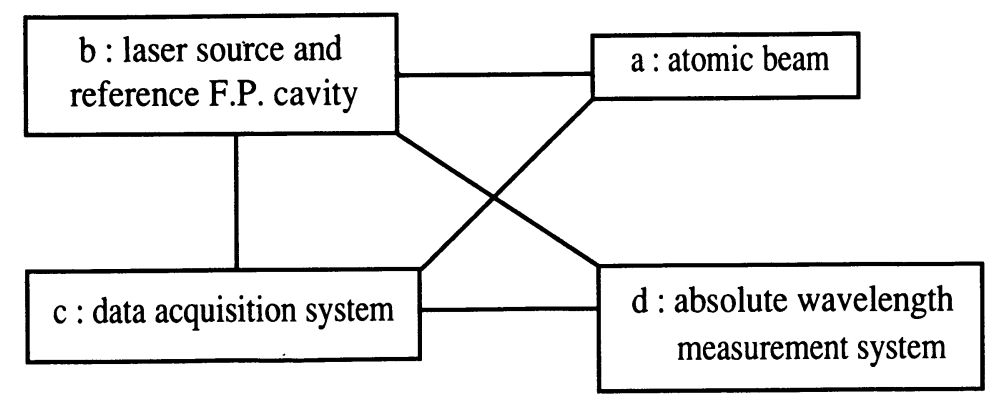

Fig. 2. - Block diagram of the whole apparatus.

data acquisition system and the wavelength measurement system. The experiment is run in two steps : i) determination of the atomic transition peak relative to a fixed reference (blocks a, b, c of Fig. 2) and ii) measurement of the absolute dye laser wavelength by comparison with the standard 
He-Ne laser (blocks b, c, d of Fig. 2). The first step involves a careful study of the line profiles which will be given in paper II; the second step relies on the interferometric technique for wavelength calibration which is one of the subjects of paper III. The details of the last portion of the apparatus (d) will also be in paper III. The quality of each component of the apparatus is important to the final goal; however, a crucial role is certainly played by the atomic beam apparatus for production and detection of the metastable $2 \mathrm{~S}$-atoms of hydrogen and deuterium.

3.1 ATOMIC BEAM. - Technical details and physical characteristics of the beam which may be of interest in different types of experiments are given elsewhere [23]. Here we mention schematically the working principles. Atomic hydrogen (or deuterium) is produced by molecule dissociation in a discharge tube; then a thermal beam of ground state atoms flows into a vacuum system which is divided in three chambers. In the first chamber, the atoms are excited to the $2 S$ state by electronic bombardment; the deviation of $\approx 20^{\circ}$ due to the inelastic collisions with the electrons is used to get a metastable beam which is collinear with the two counterpropagating laser beams as shown in figure 3. The metastable atoms, with the proper velocity direction, enter then a second chamber

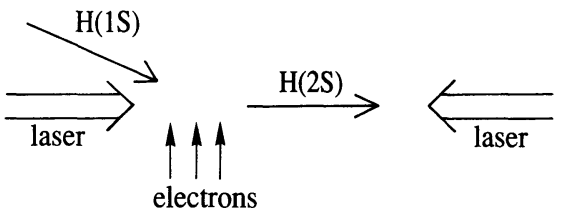

Fig. 3. - Experimental geometry of laser and atom beams.

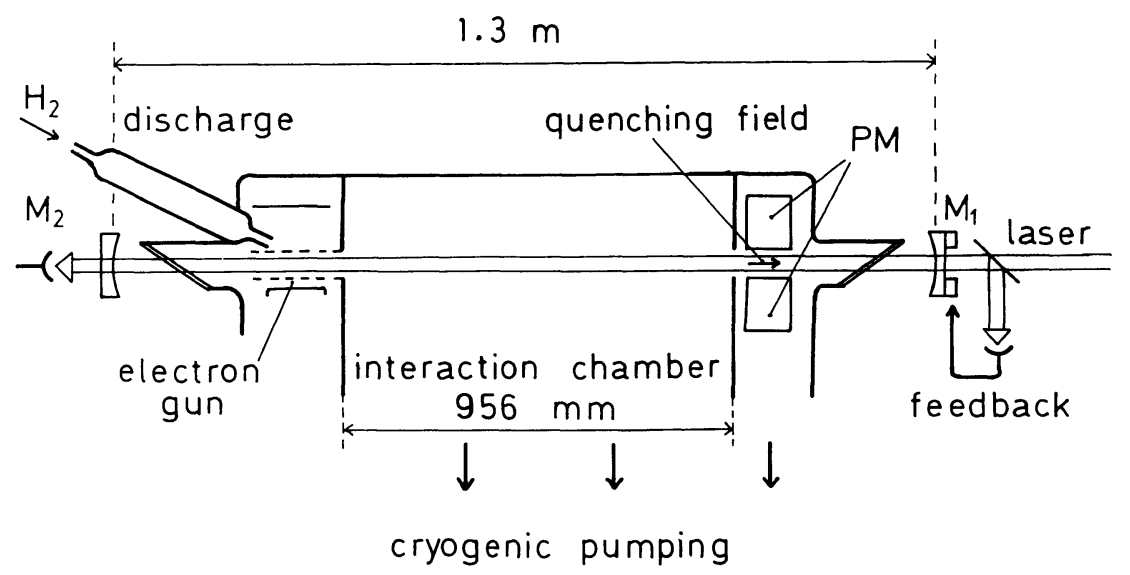

Fig. 4. - Interaction chamber and detection system.

with entrance and exit apertures consisting of two $7 \mathrm{~mm}$ diameter diaphragms (see Fig. 4). This region, where the atoms interact with the laser light, is evacuated by two cryogenic pumps and 
is carefully shielded against stray magnetic and electric fields which affect the atomic lineshape and line position. The metastable atoms are detected in a third chamber where an electric field is applied to mix the $2 S$ and $2 \mathrm{P}$ levels and the resulting Lyman- $\alpha$ fluorescence is detected by a pair of photomultipliers symmetrically positioned with respect to the atomic beam. The quenching voltage is square-wave modulated at $830 \mathrm{~Hz}$ and a phase sensitive detection, at the same frequency, is used to monitor the photomultiplier outputs. The voltage applied to each photomultiplier is adjusted in such a way that both deliver equal signals : the signal-to-noise ratio of the sum signal is then maximized. From the photomultiplier efficiencies and the detection solid angle, we can deduce the value of the metastable atom flux : $2.6 \times 10^{6} \mathrm{~s}^{-1}$. The velocity distribution of the metastable atoms has been also studied : it has been deduced from the Doppler-shifted $2 S$ 3P Balmer- $\alpha$ absorption profile recorded with a dye laser beam collinear with the metastable beam. The measured most probable velocities in the beam are $3.05 \mathrm{~km} / \mathrm{s}$ for hydrogen atoms and $1.97 \mathrm{~km} / \mathrm{s}$ for deuterium atoms.

3.2 LASER SOURCE. - The source used for the optical excitation is a home-made c.w. ring dye laser described in reference [24]. The radiation needed for the $2 S-n D(n \geq 8)$ two-photon transition is in the $730-780 \mathrm{~nm}$ range, obtainable with $\mathrm{LD} 700$ dye pumped by a $\mathrm{Kr}^{+}$laser. The dye laser delivers power up to $1 \mathrm{~W}$ in single mode operation in this wavelength range.

The frequency stabilization is done by locking the dye laser to an external $25 \mathrm{~cm}$ long FabryPerot cavity, by monitoring the reflected beam polarization according to the method of Hänsch and Couillaud [25]. An electro-optic device inserted in the dye laser cavity reduces the laser jitter to about $50 \mathrm{kHz}$.

To sweep and control the dye laser-system an additional optical device has been placed outside the optical cavity of the original laser, as shown in figure 5. The frequency reference is a homemade $\mathrm{I}_{2}-$ stabilized He-Ne laser at $633 \mathrm{~nm}$. To check the stability of this laser, the beat frequency with a second standard He-Ne laser, locked to a close $I_{2}$ molecular line, is continously monitored. A reference Fabry-Perot cavity (FPR) is locked to this etalon laser so that its length is fixed. This cavity is very stable : it consists of a $50 \mathrm{~cm}$ long zerodur rod, two silver coated mirrors, one flat and one spherical $(R=60 \mathrm{~cm})$, and a PZT, all in an evacuated box. The cavity finesse is 100 at $633 \mathrm{~nm}$ and 150 at $780 \mathrm{~nm}$. To sweep the dye laser frequency, the laser beam is split and the secondary beam enters an acousto-optic device. The frequency-shifted beam is reflected back into the acousto-optic crystal so that one of the emerging beams is shifted twice. This beam then enters the reference cavity. Its frequency is locked to the cavity length using a servo-loop acting on the dye laser external Fabry-Perot cavity (for this servo-loop, the length of the reference cavity is modulated at $5.5 \mathrm{kHz}$ ). By changing the acousto-optic modulation frequency, which is provided by a computer-controlled frequency synthesizer, we can sweep the dye laser frequency over a range of $250 \mathrm{MHz}$ centered at any desired frequency. This whole set-up allows us to control very precisely the dye laser frequency with a reproducibility of about $10^{-11}$.

To induce the two-photon $2 S-n D$ transitions with a good efficiency, we have placed the whole metastable beam apparatus inside a Fabry-Perot cavity (see Fig. 4) having its optical axis in coincidence with the metastable beam. The cavity is $1.3 \mathrm{~m}$ long and the two mirrors have $4 \mathrm{~m}$ radii of curvature so that the beam waist $w_{0}$ inside the cavity is about $600 \mu \mathrm{m}$. The transmission of the entry mirror $M_{1}$ is $1.5 \%$ and the end mirror $M_{2}$ is a high reflector. The two mirrors are mounted on PZT and the cavity length is locked to the laser wavelength by monitoring the reflected beam polarization [25]. The total laser-atom interaction length is $956 \mathrm{~mm}$, defined by the pair of apertures placed at the entrance and the exit of the second vacuum chamber.

Metastable atoms in the cavity are then submitted to an intense optical standing wave resulting from the superposition of two counterpropagating waves (power up to $50 \mathrm{~W}$ each). The light 


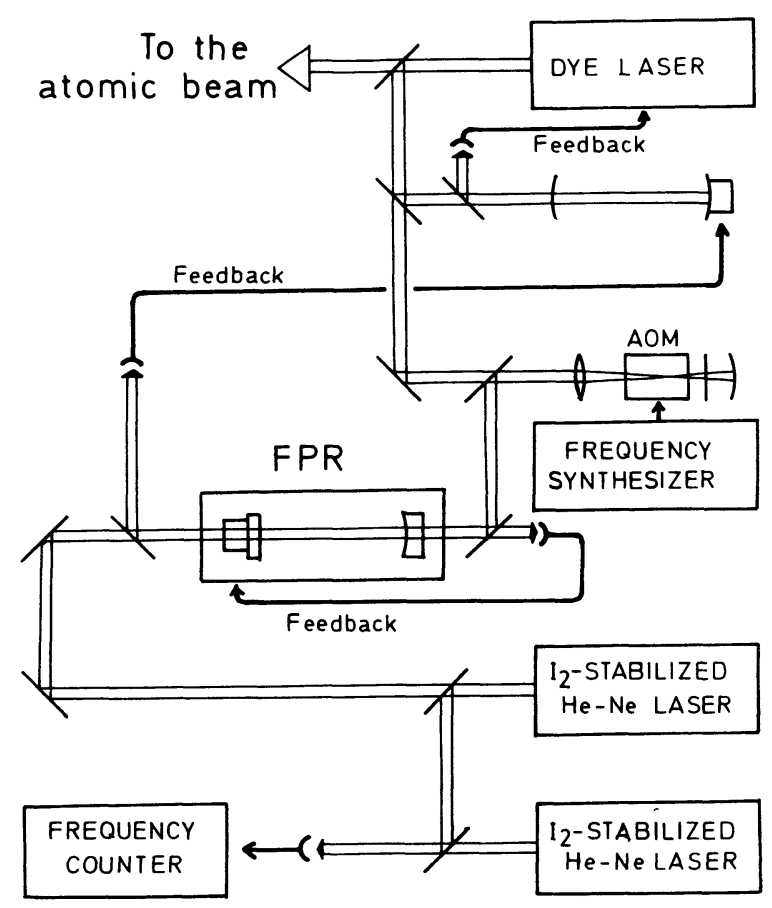

Fig. 5. - Scheme of the experimental set-up to control the dye laser frequency.

intensity inside the optical cavity is controlled by monitoring with a photodiode the small portion of the laser light which is transmitted by the cavity end mirror $\mathrm{M}_{2}$.

3.3 DATA ACQUISITION SYSTEM. - One major improvement to our original apparatus used for the 1986 measurement of $R_{\infty}$ [9] is the use of a microcomputer for the control of several parts of the equipment, and for the data acquisition and analysis. In the data acquisition we have to distinguish between the detection of the two-photon atomic transitions and the recording of all other parameters necessary to get the final result.

After optical excitation of the atomic transition, there are several methods that can be used for detection : fluorescence measurements (for example on the $\mathrm{nD}-2 \mathrm{P}$ transition), field ionization (quite efficient for $n \geq 25$ ), quenching of the $2 S$ metastable atoms. In the present work we have chosen this third method, detecting the decrease of the metastable beam intensity. When the atoms are optically excited from the $2 \mathrm{~S}$ metastable state to a $n \mathrm{D}$ Rydberg level, $95 \%$ decay to the 1S ground state through a radiative cascade, and the optical transition can be detected as a decrease of the $2 \mathrm{~S}$ population. The advantage of this method is that all optically excited atoms contribute to the observed signal, independently of their position in the beam. Indeed, figure 4 shows that the metastable atoms are produced just before entering the region of interaction with the laser and detected just after leaving it. The disadvantage is that the signal is detected over the large metastable population background which is not favourable to the signal-to-noise ratio. For the Rydberg constant determination we have used only the $2 \mathrm{~S}_{1 / 2}(F=1)-n \mathrm{D}_{5 / 2}(n=8,10,12)$ transitions because they give the more intense signals.

We have already explained how the dye laser is driven by a computer-controlled synthesizer 
which determines the absolute laser frequency with respect to a peak of the reference Fabry-Perot cavity. By sweeping the frequency of the synthesizer we can scan the dye laser over the atomic transition and detect the resulting signal on the metastable atom population. In practice, for a typical signal recording which lasts about 20 minutes (one "run"), the laser frequency is scanned ten times across the atomic resonance, alternately upwards and downwards. Simultaneously, the computer records the signal provided by the lock-in amplifier which measures the number of metastable atoms at the end of the atomic beam. Other useful data such as the beat frequency between the two etalon lasers and the light intensity transmitted by the excitation optical cavity are also collected. These data are useful to eliminate from the run the signal acquired during bad conditions of operation. Afterwards the signal is averaged over the ten scans. This procedure is illustrated
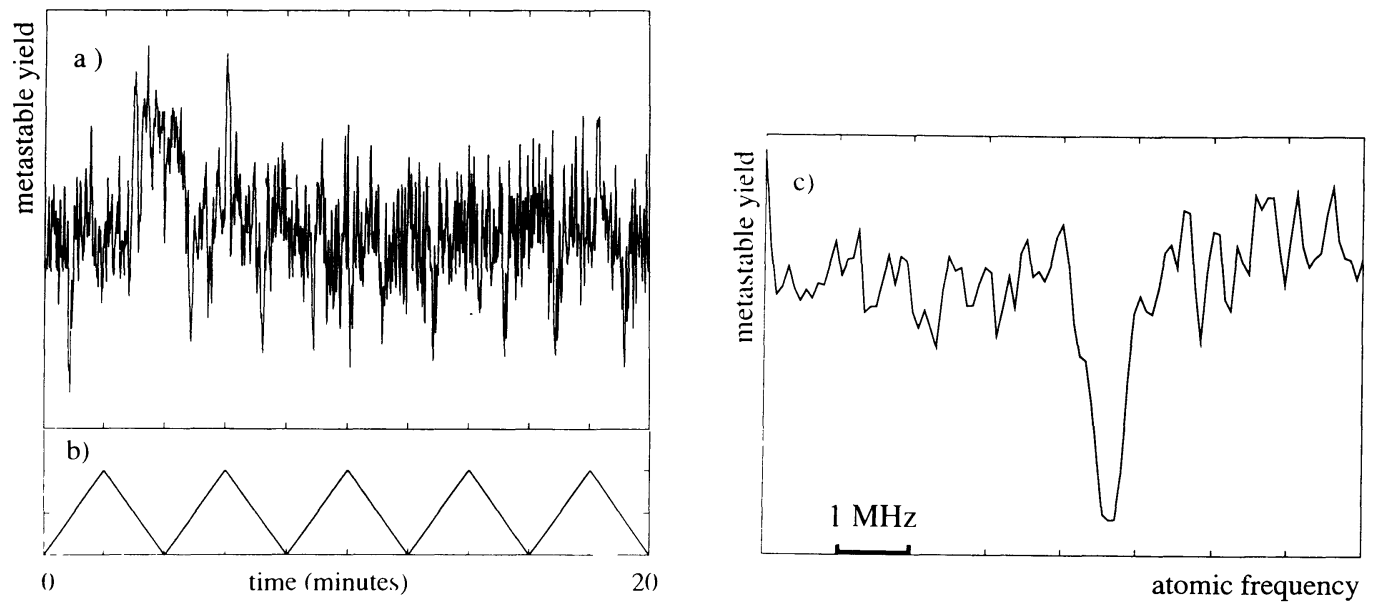

Fig. 6. - Example of an experimental run $\left(2 S_{1 / 2}-10 S_{1 / 2}\right.$ transition in deuterium): signal recorded by the lock-in amplifier (a) and laser frequency scans (b) during a run of 20 minutes; two-photon signal averaged over 10 scans versus the atomic frequency (c).

in figure 6 in the case of the $2 S_{1 / 2}-10 S_{1 / 2}$ transition in deuterium. Figure 6 a shows the metastable beam intensity and figure $6 \mathrm{~b}$ the variation of the laser frequency during the run; after the average over ten scans, the two-photon signal clearly appears, as shown in figure $6 c$.

3.4 WAVELENGTH MEASUREMENT SYSTEM. - Since this portion of the apparatus (block $d$ of Fig. 2) will be described in detail in paper III, we simply report here the basic idea of the measurement. The absolute frequency of the dye laser (and consequently the measured wavelength of the atomic transition) is determined by an interferometric comparison with the frequency of our reference $\mathrm{I}_{2}$-stabilized He-Ne laser. This laser has been compared with one of the "Institut National de Métrologie" which has been previously compared with the standard He-Ne lasers of the "Bureau International des Poids et Mesures". The key of the wavelength comparison is an etalon Fabry-Perot cavity which has been specially designed and built to match the requirements of the present experiment. 


\section{Observed signals.}

Using the method and the experimental set-up described so far, we have recorded lines corresponding to the atomic transitions listed in table I together with their amplitude. The list of table I is not exhaustive; in principle all the transitions covered by our laser source could be monitored. We have selected some of them for the determination of $R_{\infty}$ (namely those which present the largest amplitude), some others to check the limit of the experimental linewidth we can observe with this apparatus and finally some transitions with $n=20$. These high $n$ transitions, which were not accessible in our previous experiment [9] , have been now observed because in the new beam apparatus the residual stray fields have been drastically reduced. We have in fact used the $n=20$ transitions to estimate an upper limit for the Stark effect due to parasitic electric fields.

Table I. - Observed transitions and signal amplitudes (percentage decrease of the metastable beam).

\begin{tabular}{|c|c|}
\hline Transition & Maximum signal amplitude \\
\hline $\mathrm{H}: 2 \mathrm{~S}_{1 / 2}(F=1)-8 \mathrm{D}_{5 / 2}$ & $16.3 \%$ \\
$2 \mathrm{~S}_{1 / 2}(F=1)-10 \mathrm{D}_{5 / 2}$ & $13.4 \%$ \\
$2 \mathrm{~S}_{1 / 2}(F=1)-12 \mathrm{D}_{5 / 2}$ & $9.3 \%$ \\
& \\
$\mathrm{D}: 2 \mathrm{~S}_{1 / 2}(F=3 / 2)-8 \mathrm{D}_{5 / 2}$ & $17.0 \%$ \\
$2 \mathrm{~S}_{1 / 2}(F=3 / 2)-10 \mathrm{D}_{5 / 2}$ & $13.6 \%$ \\
$2 \mathrm{~S}_{1 / 2}(F=3 / 2)-12 \mathrm{D}_{5 / 2}$ & $11.3 \%$ \\
$2 \mathrm{~S}_{1 / 2}(F=3 / 2)-20 \mathrm{D}_{3 / 2,5 / 2}$ & $2.4 \%$ \\
$2 \mathrm{~S}_{1 / 2}(F=3 / 2)-10 \mathrm{~S}_{1 / 2}$ & $2.4 \%$ \\
\hline
\end{tabular}

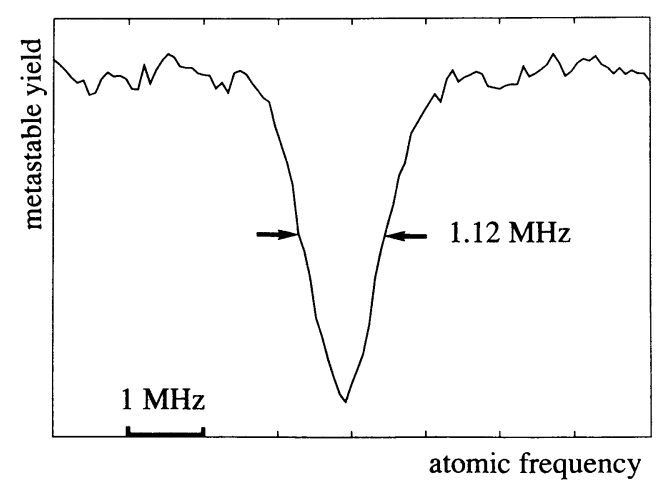

Fig. 7. - Typical two-photon transition signal, recorded as a decrease of the metastable beam intensity (example of the $2 \mathrm{~S}_{1 / 2}(F=1)-10 \mathrm{D}_{5 / 2}$ transition in hydrogen). 
An example of line used for the Rydberg constant measurement is given in figure 7 which shows a typical recording of the $2 S_{1 / 2}-10 D_{5 / 2}$ transition in hydrogen. The signal amplitude corresponds to a $9.4 \%$ decrease of the metastable beam intensity. The largest signals have been obtained for $n=8:$ their amplitude is up to $17 \%$ and the corresponding signal-to-noise ratio about 60 . The experimental linewidth is related to the natural width of the upper state involved in the transition and to several other parameters. In figure 7, the observed linewidth is $1.1 \mathrm{MHz}$ in terms of total two-photon transition frequency. The natural width of the $10 \mathrm{D}$ level is $296 \mathrm{kHz}$ only; as is described in papers II and III, the main cause of signal broadening is the inhomogeneous lightshift that atoms undergo in the laser beams. The observed relative linewidth is here $1.6 \times 10^{-9}$. We have in fact observed linewidths as narrow as $600 \mathrm{kHz}\left(2 S_{1 / 2}-10 S_{1 / 2}\right.$ transition of Fig. 6) corresponding to a relative linewidth of $7.6 \times 10^{-10}$.

\section{Conclusion.}

We have described the method and the apparatus used to investigate the Doppler-free two-photon transitions $2 S-n S$ and $2 S-n D(n \geq 8)$ in atomic hydrogen and deuterium. The main characteristics of our experiment are the choice of narrow resonances (a few parts in $10^{10}$ ) and the actual ability to observe them (about 1 part in $10^{9}$ ). The following two papers describe other aspects of the experiment.

\section{Acknowledgements.}

The authors are indebted to Professor B. Cagnac for much fruitful advice during this experiment. They also thank Professor N.K. Rahman for reading the manuscript. This work is partially supported by the Bureau National de Métrologie. One of us (J.C.G.) is grateful to the Brazilian Financial Agency CAPES for a scholarship.

\section{References}

[1] Biraben F., Garreau J. C., JUlien L. and Allegrini M., Phys. Rev. Lett. 62 (1989) 621.

[2] See for example SERIES G. W., The Spectrum of Atomic Hydrogen, Advances (World Scientific Publishing) 1988.

[3] MCINTYRE D. H. and HĀNSCH T. W., Metrologia 25 (1988) 61.

[4] COHEN E. R. and TAYLOR B. N., Rev. Mod. Phys. 59 (1987) 1121.

[5] PETLEY B. W., The Fundamental Physical Constants and the Frontier of Measurement (Adam Hilger Ltd, Bristol) 1985.

[6 BARLETT D. F. and LOGL S., Phys. Rev. Lett. 61 (1988) 2285.

[7] JULIEN L., BIRABEN F. and CAGNAC B., Bulletin du B.N.M. 66 (1986) 31.

[8] ZHAO P., LICHTEN W., LAYER H. P. and BERGQUIST J. C., in Laser Spectroscopy VIII, Eds. W. Persson and S. Svanberg (Springer Verlag, Heidelberg) 1987, p.12.

[9] BIRABEN F., GARREAU J. C. and JULIEN L., Europhys. Lett. 2 (1986) 925.

[10] ZhaO P., Lichten W., LAYER H. P. and BERGQUIST J. C., Phys. Rev. A34 (1986) 5138.

[11] ZHAO P., LICHTEN W., LAYER H. P. and BERGQUIST J. C., Phys. Rev. Lett. 58 (1987) 1293.

[12] Beausoleil R. G., McInTyRe D. H., Foot C. J., Hildum E. A., Couillaud B. and Hânsch T. W., Phys. Rev. A35 (1987) 4878.

[13] Boshier M. G., BAIRd P. E. G., FOOT C. J., Hinds E. A., Plimmer M. D., STACEy D. N., Swan J. B., TATE D. A., WARRINGTON D. M. and WOODGATE G. K., Nature 330 (1987) 463; Phys. Rev. A40 (1989) 6169. 
[14] Hänsch T. W., NAyfeh M. H., LeE S. A., CURRY S. M. and Shahin I. S., Phys. Rev. Lett. 32 (1974) 1336.

[15] Jennings D. A., Pollock C. R., Petersen F. R., Drullinger R. E., Evenson K. M., Wells J. S., HALL J. L.and LAYER H. P., Opt.Lett. 8 (1983) 136.

[16 AMIN S. R., CALDWELl C.D. and LICHTEN W., Phys. Rev. Lett. 47 (1981) 1234.

[17] LAMB W. E. and ReTHERFoRd R. C., Phys. Rev. 81 (1951) 222.

[18] Biraben F. and Julien L., Opt. Comm. 53, 319 (1985);

Biraben F. and Julien L., C.R. Hebdo. Acad. Sci. Paris 300 (1985) 161.

[19] CAGNAC B., GRYNBERg G. and BiRABEN F., J. Phys. France 34 (1973) 845.

[20] PAL'ChIKOV V. G., SOKOLOV Yu. L. and YAKOVLEV V. P., JETP Lett. 38, (1983) 418 ; Metrologia 21 (1985) 99.

[21] LundeEn S. R. and PiPkin F. M., Phys. Rev. Lett. 46 (1981) 232.

[22] ERICKSON G. W. and GROTCH H., Phys. Rev. Lett. 60 (1988) 2611; 63 (1989) 1326.

[23] BIRABEn F., GARREAU J.C., JULIEN L. and ALlEGRINI M., Rev. Sci. Instrum. 61 (1990) 1468.

[24] Biraben F. and LaBastie P., Opt. Commun. 41 (1982) 49.

[25] HänSCH T. W. and Couillaud B., Opt. Commun. 35 (1980) 441.

Cet article a êté imprimé avec le Macro Package "Editions de Physique Avril 1990". 